\title{
MODIFICATION OF THE SPONTANEOUS EMISSION OF DYE MOLECULES IN PHOTONIC CRYSTALS*
}

\author{
E.P. Petrov ${ }^{a} \dagger$, V.N. Bogomolov ${ }^{b}$, I.I. Kalosha ${ }^{c}$ and S.V. GaPonenKo ${ }^{c}$ \\ ${ }^{a}$ B.I. Stepanov Institute of Physics, National Academy of Sciences of Belarus \\ F. Skaryna Ave. 68, Minsk 220072, Belarus \\ ${ }^{b}$ A.F. Ioffe Physico-Technical Institute, Russian Academy of Sciences \\ Politekhnicheskaya St. 26, St.-Petersburg 194021, Russia \\ ${ }^{c}$ Institute of Molecular and Atomic Physics, National Academy of Sciences of Belarus \\ F. Skaryna Ave. 70, Minsk 220072, Belarus
}

(Received July 23, 1998)

\begin{abstract}
Modification of the spontaneous emission of dye molecules embedded in artificial opals exhibiting stop bands in the visible range is observed. Molecules embedded in artificial opals show dips in spontaneous emission spectra and modified fluorescence decay kinetics. Results are interpreted in terms of redistribution of the photon density of states. Effects of the multiple reabsorption and reemission of fluorescence photons and interaction of dye molecules with the silica surface are discussed.
\end{abstract}

PACS numbers: $42.50 .-\mathrm{p}$

\section{Introduction}

The concept of the spontaneous emission control by means of modification of the photon density of states (DOS) in mesoscopic structures [1] has gained considerable interest during the last decade $[2,3]$. The phenomenon is a subject of extensive theoretical investigations (see, e.g., [4-7] and references therein). Experiments in this field include, but are not limited to, studies of modification of the spontaneous emission in the vicinity of a dielectric interface [8], in dielectric slabs [9-13], Fabri-Perot microcavities [14-17], semiconductor nanostructures [18, 19], liquid microdroplets [20], water-in-oil micelles [21], phospholipid bilayers [22, 23], and cell membranes [24].

*The results of this paper were initially presented at The Jabtoriski Centennial Conference on Luminescence and Photophysics, July 23-27, 1998, Torun', Poland.

†Corresponding author. E-mail: petrov@ifanbel.bas-net.by; phone: +375 017 2840970; fax: +3750172840879. 
Another approach to the modification of the photon DOS implies fabrication of optically inhomogeneous structures consisting of microscopic light scatterers either arranged in a three-dimensional (3D) periodic lattice (photonic crystal) or randomly distributed in space. The ideas of the photonic band engineering have been advanced more than a decade ago $[25,26]$ and have been investigated in a number of theoretical studies (see, e.g., $[2,3,27,28]$ and references therein). Depending on parameters of scatterers (size, shape, and dielectric function) and type of their spatial arrangement (volume fraction and symmetry), coherent effects in light scattering may result in either localization of light waves or the development of a spectral range within which the propagation of light is forbidden (photonic band gap). These phenomena are similar to the well-known features of electrons in disordered and crystalline solids, respectively, except for the fact that in the case of electrons, the phenomena are essentially quantum mechanical, whereas they have a purely classical origin in the case of electromagnetic waves.

Until recently, studies of the effect of 3D photonic band gap structures on the spontaneous emission in the visible range have been restricted to experiments with dye molecules embedded in ordered aqueous suspensions of polystyrene microspheres, for which a slowing down of the spontaneous emission has been reported by Martorell and Lawandy [29]. However, a comprehensive investigation of the

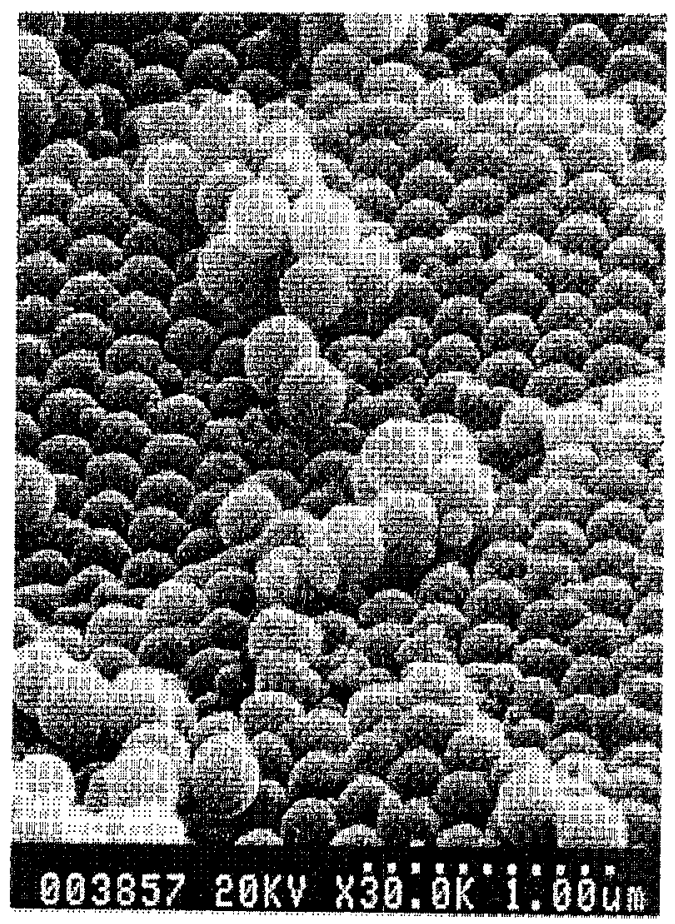

Fig. 1. Electronic microphotograph of opal sample. The scale of the image is given by the row of dots in the lower black panel: neighboring dots are separated by a distance of $100 \mathrm{~nm}$. 
same system carried out later by Tong et al. [30] has shown that intermolecular interactions of dye molecules with the surface of polystyrene microspheres and a strong overlap of absorption and fluorescence spectra of the dye were responsible for a major fraction of the change in the measured lifetime. In our recent letter [31] we reported on a modification of the spontaneous emission of dye molecules embedded in polymer-filled artificial opal, which resulted in appearance of a dip in the fluorescence emission spectrum and nonexponential spontaneous decay kinetics containing both inhibited and accelerated decay components.

Artificial opals are rigid solid-state colloidal crystals consisting of submicrometer-sized amorphous silica globules. During the synthesis of opals, silica globules are self-organized in the face-centered cubic (fcc) lattice (Fig. 1). A solid experimental evidence in support of the fcc structure of opals has recently been presented in Ref. [32]. The fcc arrangement of silica globules is favorable to the formation of a pronounced photonic stop band in the visible range which extends over a region of angles and manifests itself in optical transmission and reflection experiments [33-36]. Voids between globules constituting opal are interconnected and thus form a three-dimensional network that can be filled with liquids and solids and provides an opportunity to embed light-emitting species in opals [31, 34, 35]. The photonic stop band in opals can be substantially enhanced by filling the network of interglobule voids with high-refracting substances [33, 34, 36].

In this article, we present a more detailed account of our previous investigation of fluorescence of dye molecules in polymer-filled opal [31] and compare the results with our experimental data on the fluorescence of dye molecules embedded in dry opal samples.

\section{Experimental}

Artificial opals were synthesized from a sol of highly monodispersed porous silica globules with the diameter ranging from 200 to $300 \mathrm{~nm}$ by means of hydrothermal treatment and annealing as described elsewhere [37].

Optical transmission of opal samples was measured on a CCD-array-based spectrometer [38] modified for optical transmission measurements.

Two sets of fluorescence experiments were carried out in the present work. In the first set, fluorescence of Rhodamine 6G (R6G) dye embedded in dry opal samples was investigated. The dye was introduced by impregnating opal samples with $\mathrm{a} \approx 10^{-4} \mathrm{M}$ solution of $\mathrm{R} 6 \mathrm{G}$ in ethanol and subsequent drying of the solvent at $40^{\circ} \mathrm{C}$. In the second set of experiments, we investigated fluorescence of 1,8-naphthoylene- $1^{\prime}, 2^{\prime}$-benzimidazole (7H-benzimidazo[2,1-a]benz[de]isoquinolin-7-one) dye referred to in what follows as NBIA dissolved in poly(methyl methacrylate) (PMMA) filling voids between the globules constituting opal. The sample was fabricated by impregnating opal with an NBIA solution in methyl methacrylate with the subsequent polymerization. An optically thick $(\approx 0.5 \mathrm{~mm})$ polymer film prepared from the same solution was used as a reference sample. The resulting concentration of NBIA in PMMA was about $10^{-5} \mathrm{M}$.

Fluorescence spectra and intensity decays were measured on an automated laser spectrometer described in detail elsewhere [39]. A TEA $\mathrm{N}_{2}$ laser was used for excitation at $337.1 \mathrm{~nm}$. Fluorescence was collected at different angles with respect 
to the normal to the surface of the sample within a solid angle of $0.09 \pi$ sr. Fluorescence decays collected in 1000 time chainnels with $0.1 \mathrm{~ns} /$ channel were analyzed using both the conventional one- to three-exponential nonlinear fitting method and the decay time distribution analysis technique (see, e.g., [40, 41]). In the present work the model-free analysis of fluorescence decay traces was carried out using a regularized method proposed by Petrov [24, 42]. In the analysis, a fluorescence decay is modeled by an exponential series $I(t)=\sum_{i=1}^{n} f_{i} \exp \left(-t / \tau_{i}\right)$ with fixed decay times $\tau_{i}$ and varied pre-exponential factors $f_{i} \cdot n=100$ exponential terms with decay times logarithmically spaced within the interval $0.05 \ldots 50.0$ ns were included in the analysis. In the present investigation, distributions of fluorescence decay times are presented as plots of steady-state intensities $f_{i} \tau_{i}$ vs. decay times $\tau_{i}$. Since no fluorescence build-up is expected in the system under investigation, fluorescence decay time distributions wer" - mputed subject to nonnegative constraints.

\section{Results and discussion}

\subsection{Experiments with dry opals}

In the first set of experiments, we investigated fluorescence of R6G dye embedded in dry opal samples. Two opal samples were used in this investigation: one sample exhibited a stop band at $\approx 550 \mathrm{~nm}$, i.e. within the limits of the R6G fluorescence spectrum, and another sample had a stop band located well to the blue compared to the R6G spontaneous emission. The latter sample should not affect the photon DOS within the spectral range of the dye fluorescence and therefore it is referred to as the "vacuum" sample. The spontaneous emission of R6G is modified by the opal whose stop band overlaps the R6G spontaneous emission spectrum, which manifests itself as a dip in the fluorescence spectrum of the dye (Fig. 2). Since the stop band is angular-dependent, the position of the dip also depends on the observation angle.

Fluorescence kinetics of R6G in the two opal samples was found to follow closely the single-exponential decay law (Fig. 3). The fluorescence from the sample whose stop band overlapped the dye emission (mean lifetime $\bar{\tau}=4.9 \mathrm{~ns}$ ) was found to decay slower by a factor of 1.5 slower than the fluorescence of R6G in the "vacuum" sample ( $\bar{\tau}=3.3 \mathrm{~ns})$. This can be attributed to the effect of the modified photon DOS in the opal sample on the spontaneous emission rate. However, the R6G dye is famous for its strongly overlapping absorption and fluorescence spectra. This circumstance, especially at considerable concentrations of the dye, can result in the multiple reabsorption and reemission of fluorescence, which leads to an increase in the apparent fluorescence lifetime (see, e.g., [43]). Recall that the stop bands in opals are due to the Bragg reflection of light from the periodically arranged optical inhomogeneities. Therefore, the effective path length of fluorescence photons is larger in the sample whose stop band overlaps the dye fluorescence. This leads to an increase in the probability of reabsorption of fluorescence photons by unexcited dye molecules, and therefore is equivalent to an increase in the dye concentration in a homogeneous medium. It is therefore instructive to compare our results with the results of Alfano et al. [44] who have 


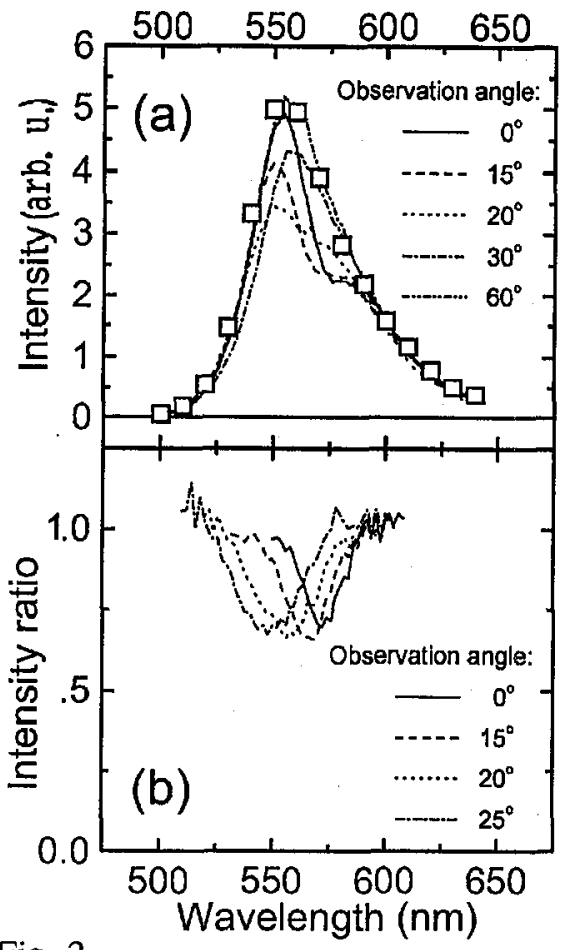

Fig. 2

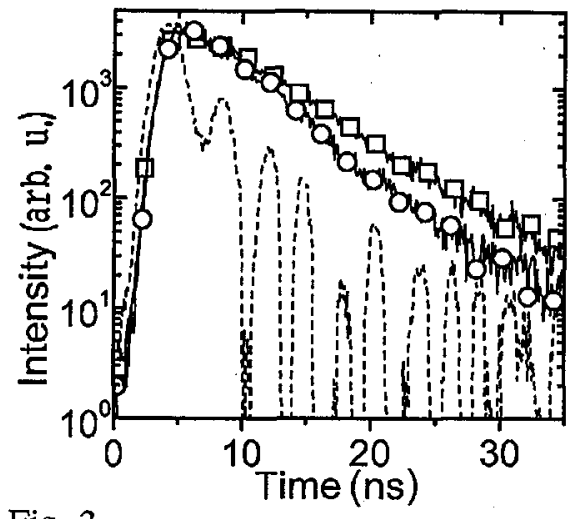

Fig. 3

Fig. 2. Fluorescence emission spectra of R6G dye molecules embedded in dry opal samples. Panel (a): fluorescence spectra of R6G in opal sample with the stop band overlapping the fluorescence band measured at different observation angles with respect to the normal to the surface of the sample. Open squares show fluorescence spectrum of the dye from the sample with the stop band located well to the blue from the fluorescence band ("vacuum" spectrum). Panel (b): modified fluorescence emission spectra divided by the "vacuum" spectrum.

Fig. 3. Experimentally detected fluorescence decay kinetics of R6G dye molecules embedded in dry opal samples: fluorescence kinetics recorded for the sample with the stop band overlapping (- - -) and with the stop band located outside (-0-) the fluorescence emission band, and the apparatus response function of the fluorometer (-- -). Excitation wavelength $337.1 \mathrm{~nm}$, emission wavelength $575 \mathrm{~nm}$.

observed an increase in the fluorescence lifetime of $\mathrm{R} 6 \mathrm{G}$ in ethanol from $3.1 \mathrm{~ns}$ at low concentrations $\left(\leq 10^{-5} \mathrm{M}\right)$ to $4.8 \mathrm{~ns}$ at the concentration of $2.0 \times 10^{-4} \mathrm{M}$. We should also mention that different synthesis protocols were followed to produce opals with different positions of optical stop bands, and thus the opal samples could have different properties of the surface on the molecular scale. Therefore, different amounts of the dye could have been adsorbed on the surface of silica globules in different opal samples. Moreover, the state of the silica surface is known to affect substantially fluorescence lifetimes and quantum yields of dye molecules adsorbed [45], which also cannot be ruled out in the case under consideration. 
Thus, in the experiments with R6G dye molecules embedded in dry opals, an increase in the fluorescence lifetime of the dye in the sample whose photonic stop band overlapped the fluorescence spectrum of $R 6 \mathrm{G}$ is attributed to a joint effect of the modification of the photon DOS by the dielectric lattice and multiple reabsorption and reemission of fluorescence photons due to strongly overlapping absorption and emission spectra of the dye.

\subsection{Experiments with polymer-filled opals}

To eliminate the effect of the multiple reabsorption and reemission of fluorescence photons on the fluorescence lifetime one should choose dye molecules with the minimum overlap of fluorescence and absorption spectra. In addition, measures should be undertaken to avoid uncontrollable interactions of dye molecules with the silica surface. Therefore, one should use dye molecules with a substantial Stokes loss which would be isolated from the silica surface. It has been found that the solution of the NBIA dye in PMMA satisfies these criteria. The NBIA dye has a high quantum yield and substantial Stokes loss in nonpolar solvents [46], and the PMMA matrix isolates dye molecules from uncontrollable interactions with the silica and provides an identical environment for dye molecules. In addition, the polymer provides a reasonably high dielectric contrast between the filler and silica globules: due to the porous structure, silica globules have a refractive index of $\approx 1.3[33,34]$, whereas the refractive index of PMMA is 1.490 .

Thus, a solid-state photonic crystal was fabricated by developing a 3D polymer network filling voids between close-packed spherical globules with a lower refractive index. Due to the fact that the globules form the fcc lattice, the volume fraction of the high-refracting network is about $25 \%$. Numerical simulations [47-49] have shown that the network topology of the high-refractive material constituting a photonic crystal is more favorable to the formation of gaps in the photon DOS, and thus is more favorable to modification of the spontaneous emission of species embedded in photonic crystals. Optical transmission measurements show that the stop band formed in the polymer-filled opal manifests itself as a dip in the optical transmission spectrum (Fig. 4) lying within the fluorescence emission band of the NBIA band.

Fluorescence spectra of NBIA in a separately standing polymer film and polymer network structure filling interconnected voids between globules constituting opal are presented in Fig. 5a along with the fluorescence excitation spectrum of NBIA in PMMA. It is evident that the spontaneous emission is modified in polymer-filled opal: a dip in the emission spectrum appears whose position is close to that of the dip in the optical transmission spectrum of the polymer-filled opal sample. The ratio of emission intensities of the dye in systems with the modified (opal sample) and "vacuum" (polymer film) photon densities of states is shown in Fig. 5b.

Fluorescence kinetics of NBIA in the PMMA film and polymer-filled opal are shown in Fig. 6. It has been found that the dye emission is contaminated by a relatively short-lived ( $\approx 1$ ns lifetime) fluorescence coming from impurities in the polymer and silica, and therefore, the fluorescence decays should be analyzed carefully. The substantial difference between the dye and impurity fluorescence 


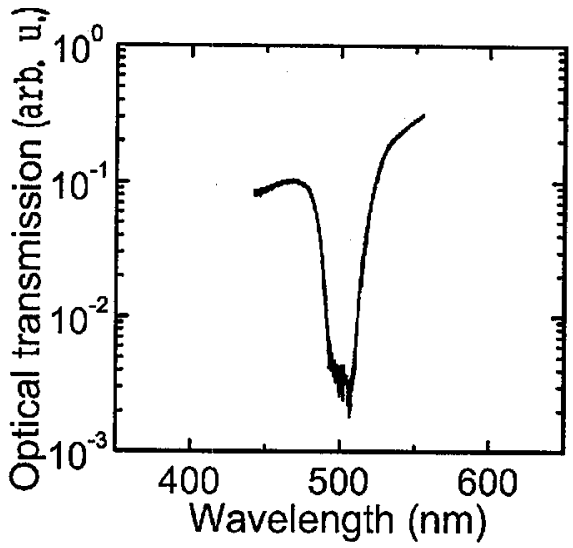

Fig. 4. Optical transmission spectrum of opal sample with interglobule voids filled with poly(methyl methacrylate).

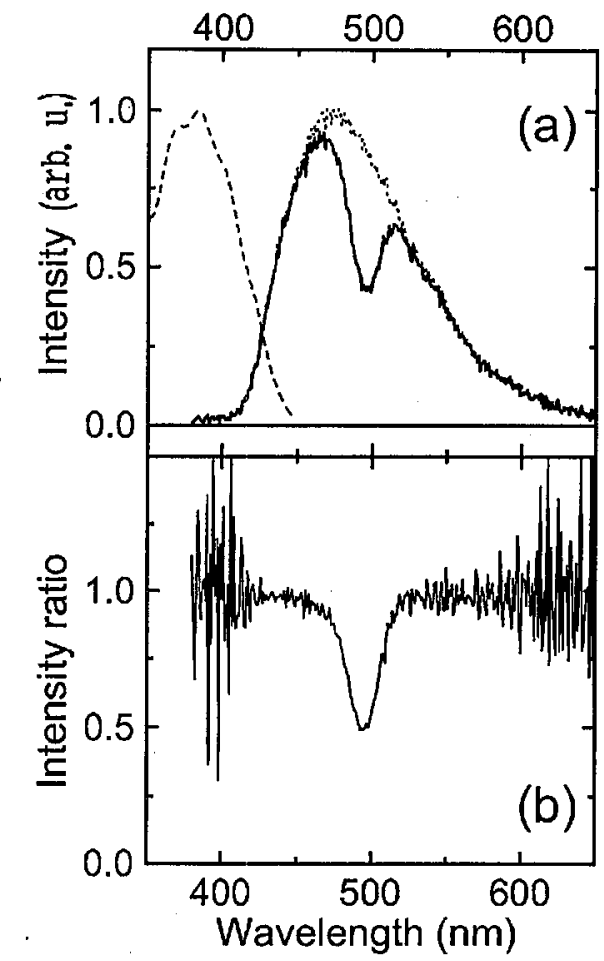

Fig. 5. (a) Fluorescence emission spectrum of NBIA solution in PMMA filling voids in opal (-), and fluorescence emission (..) and excitation (- - ) spectra of NBIA in a free-standing PMMA film (impurity fluorescence subtracted). Fluorescence was excited at $337.1 \mathrm{~nm}$, and the excitation spectrum was detected at $500 \mathrm{~nm}$. (b) Ratio of fluorescence intensities of the NBIA solution in PMMA in the opal sample and free-standing film. 


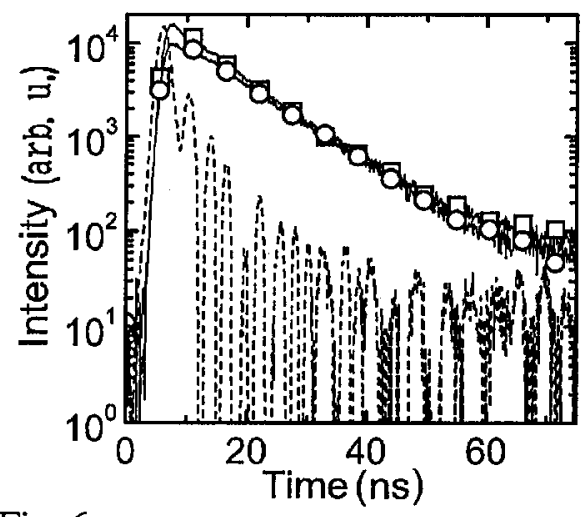

Fig. 6

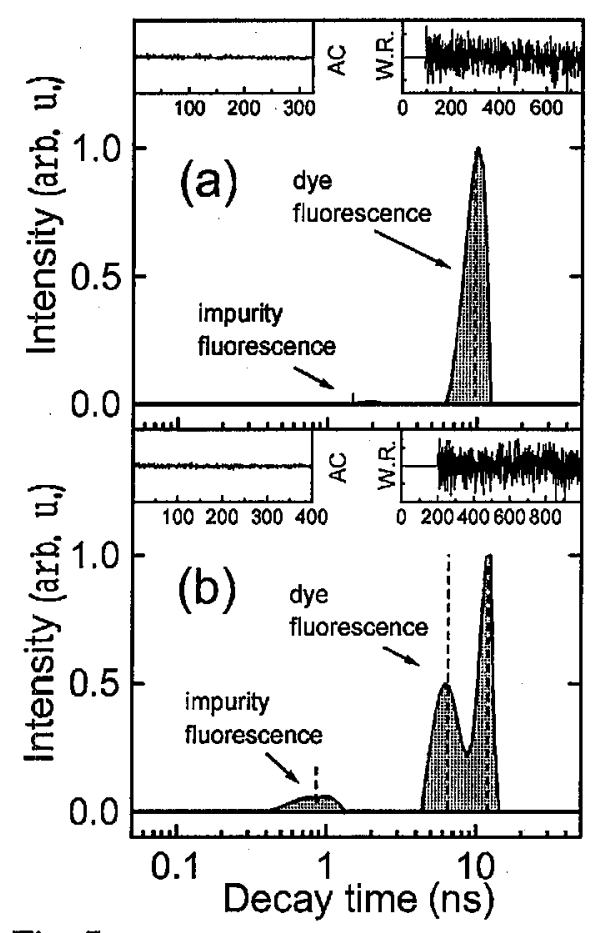

Fig. 7

Fig. 6. Experimentally detected fluorescence decay kinetics of NBIA dye molecules in the polymer-filled opal sample (-口-) and in the reference polymer film (-o-), and the apparatus response function of the fluorometer (- -). Excitation wavelength $337.1 \mathrm{~nm}$, emission wavelength $510 \mathrm{~nm}$.

Fig. 7. Fluorescence decay time distributions of NBIA recovered from kinetics presented in Fig. 5: (a) in a free-standing polymer film and (b) in polymer network filling voids in opal. Results of (a) two- and (b) three-exponential analysis of the same kinetics are shown by dashed lines. The insets show plots of weighted residuals (WR) and their autocorrelation function $(\mathrm{AC})$.

lifetimes made it possible to extract information on the excited-state decay of the dye in the samples. It should be noted that even a visual inspection of the fluorescence decay traces shows that the fluorescence kinetics of the dye embedded in the photonic crystal is more complex than the fluorescence kinetics of the reference polymer film. Results of a quantitative analysis of the fluorescence decay traces by the conventional two- and three-exponential fitting and the decay time distribution technique are presented in Fig. 7 along with weighted residuals and their autocorrelation functions [50] showing the goodness of fit by the decay time distribution model to the experimental kinetics. The analysis shows that the excited-state decay of NBIA is exponential in the reference polymer film (Fig. 7a), whereas both accelerated and inhibited decay components have been found in the spontaneous emission kinetics of dye molecules embedded in the polymer-filled opal (Fig. 7b). 
This somewhat unexpected result gains a reasonable explanation in terms of a redistribution of the photon DOS in the photonic crystal. According to the sum rule for the spontaneous emission derived by Barnett and Loudon [51], any optically inhomogeneous environment just redistributes the spontaneous radiation power over the spectrum and directions, which also applies to a photonic crystal. Indeed, numerical simulations of the dipole emission in a photonic crystal [52] have shown that the dipole radiative power undergoes a spectral and angular redistribution compared to the free space and depends on the position and orientation of the emitter with respect to an elementary crystal cell. In addition, one should take into account the fact that the fluorescence spectrum of the dye used in the investigation is substantially wider than the photonic stop band. Theoretical investigations by Mogilevtsev and Kilin [53] have shown that either acceleration or inhibition of the spontaneous decay of a dye molecule with a wide fluorescence spectrum can be observed in a photonic crystal with a band gap narrower than the spontaneous emission band, depending on the mutual spectral position of the band gap and the fluorescence spectrum. In the system under investigation, the polymer-filled opal shows an angular-dependent stop band, and since dye molecules embedded therein assume random orientations, each molecule "senses" different photon density of states corresponding to its orientation and position with respect to the elementary crystal cell, which explains the presence of both accelerated and inhibited components in the spontaneous emission decay observed in the experiment.

Thus, two competitive processes of inhibition and acceleration of the spontaneous emission due to a redistribution of the photon density of states contribute to the observed fluorescence kinetics of the NBIA dye embedded in an opal sample filled with PMMA, which manifests itself as a nonexponential excited-state decay of the dye.

\section{Conclusions}

In conclusion, we observed modification of the spontaneous emission of dye molecules in artificial opals, which manifested itself as dips in fluorescence emission spectra and modification of the spontaneous decay laws.

The observation of the pure inhibition of the spontaneous emission is expected for narrow-band emitting species, e.g., rare-earth ions and semiconductor nanocrystals, for which effects connected with the redistribution of the photon DOS across the spontaneous emission band can be avoided. At the same time, spontaneous emission and absorption spectra of photon DOS probes should have a minimum overlap to avoid effects of nultiple reabsorption and reemission of spontaneously emitted photons on the experimentally observed fluorescence decay time.

\section{Acknowledgments}

The work was supported by the INTAS Project, grant \#96-0438. 


\section{References}

[1] E.M. Purcell, Phys. Rev. 69, 681 (1946).

[2] Photonic Bandgaps and Localization, Ed. C.M. Soukoulis, Plenum Press, New York 1993.

[3] Confined Electrons and Photons: New Physics and Applications, Eds. E. Burstein, C. Weisbuch, Plenum Press, New York 1995.

[4] V.P. Bykov, Radiation of Atoms in a Resonant Environment, World Scientific, Singapore 1993.

[5] W. Lukosz, Phys. Rev. B 22, 3030 (1980).

[6] H. Khosravi, R. Loudon, Proc. R. Soc. Lond. A 433, 337 (1991); 436, 373 (1991).

[7] H.P. Urbàch, G.L.J.A. Rikken, Phys. Rev. A 57, 3913 (1998).

[8] J.K. Trautman, J.J. Macklin, T.D. Harris, L.E. Brus, Science 272, 255 (1996).

[9] K.H. Drexhage, in: Progress in Optics, Ed. E. Wolf, Vol. XII, North-Holland, Amsterdam 1974.

[10] R.E. Kunz, W. Lukosz, Phys. Rev. B 21, 4814 (1980).

[11] E. Yablonovitch, T.J. Gmitter, R. Bhat, Phys. Rev. Lett. 61, 2546 (1988).

[12] F. De Martini, M. Marocco, P. Mataloni, L. Crescentini, R. Loudon, Phys. Rev. A 43, 2480 (1991).

[13] G.L.J.A. Rikken, Phys. Rev. A 51, 4906 (1995).

[14] A.N. Rubinov, V.I. Nikolaev, Izv. Akad. Nauk SSSR, Ser. Fiz. 34, 1308 (1970).

[15] F. De Martini, G. Innocenti, G.R. Jakobowitz, P. Mataloni, Phys. Rev. Lett. 59, 2955 (1987).

[16] A.M. Vredenberg, N.E.J. Hunt, E.F. Schubert, D.C. Jacobson, J.M. Poate, G.J. Zydzik, Phys. Rev. Lett. 71, 517 (1993).

[17] V. Pellegrini, A. Tredicucci, C. Mazzoleni, L. Pavesi, Phys. Rev. B 52, R14328 (1995).

[18] M.D. Tocci, M. Scalora, M.J. Bloemer, J.P. Dowling, C.M. Bowden, Phys. Rev. A 53, 2799 (1996).

[19] J.P. Reithmaier, M. Rohner, H. Zull, F. Schafer, A. Forchel, P.A. Knipp, T.L. Reinecke, Phys. Rev. Lett. 78, 378 (1997).

[20] M.D. Barnes, W.B. Whitten, S. Arnold, J.M. Ramsey, J. Chem. Phys. 97, 7842 (1992).

[21] P. Lavallard, M. Rosenbauer, T. Gacoin, Phys. Rev. A 54, 5450 (1996).

[22] D. Toptygin, L. Brand, Biophys. Chem. 48, 205 (1993).

[23] M.M.G. Krishna, N. Periasamy, J. Fluorescence 8, 81 (1998).

[24] E.P. Petrov, J.V. Kruchenok, A.N. Rubinov, J. Fluorescence, to be published.

[25] E. Yablonovitch, Phys. Rev. Lett. 58, 2059 (1987).

[26] S. John, Phys. Rev. Lett. 58, 2486 (1987).

[27] Special Issue on Photonic Band Structures [J. Mod. Opt. 41, No. 2 (1994)].

[28] G. Kweon, N.M. Lawandy, Opt. Commun. 118, 388 (1995).

[29] J. Martorell, N.M, Lawandy, Phys. Rev. Lett. 65, 187 (1990).

[30] B.Y. Tong, P.K. John, Y.-T. Zhu, Y.S. Liu, S.K. Wong, W.R. Ware, J. Opt. Soc. Am. B 10, 356 (1993). 
[31] E.P. Petrov, V.N. Bogomolov, I.I. Kalosha, S.V. Gaponenko, Phys. Rev. Lett. 81, 77 . (1998).

[32] H. Miguez, F. Meseguer, C. López, A. Mifsud, J.S. Moya, L. Vázquez, Langmuir 19, 6009 (1997).

[33] V.N. Bogomolov, S.V. Gaponenko, A.M. Kapitonov, A.V. Prokofiev, A.N. Ponyavina, N.I. Silvanovich, S.M. Samoilovich, Appl. Phys. A 63, 613 (1996).

[34] V.N. Bogomolov, S.V. Gaponenko, I.N. Germanenko, A.M. Kapitonov, E.P. Petrov, N.V. Gaponenko, A.V. Prokofiev, A.N. Ponyavina, N.I. Silvanovich, S.M. Samoilovich, Phys. Rev. E 55, 7619 (1997):

[35] V.N. Bogomolov, A.V. Prokofiev, S.M. Samoilovich, E.P. Petrov, A.M. Kapitonov, S.V. Gaponenko, J. Lumin. 72-74, 391 (1997).

[36] A.M. Kapitonov, N.V. Gaponenko, V.N. Bogomolov, A.V. Prokofiev, S.M. Samoilovich, S.V. Gaponenko, Phys. Status Solidi A 165, 119 (1998).

[37] V.N. Bogomolov, T.M. Pavlova, Semiconductors 29, 428 (1995).

[38] I.N. Germanenko, A.M. Kapitonov, A.P. Byck, A.M. Aksyonov, I.I. Kravtsevich, S.V. Gaponenko, in: ICONO'95: Nonlinear Spectroscopy and Ultrafast Phenomena, Eds. V.V. Shuvalov, A.M. Zheltikov, Proc. SPIE, Vol. 2797, SPIE, Bellingham, WA 1996, p. 81.

[39] N.A. Nemkovich, A.S. Kozlovsky, A.N. Rubinov, Yu.V. Zvinevich, in: Advances in Fluorescence Sensing Technology II, Ed. J.R. Lakowicz, Proc. SPIE, Vol. 2388, SPIE, Bellingham, WA 1995, p. 347.

[40] A. Siemiarczuk, B.D. Wagner, W.R. Ware, J. Phys. Chem. 94, 1661 (1990).

[41] D.M. Gakamsky, A.A. Goldin, E.P. Petrov, A.N. Rubinov, Biophys. Chem. 44, 47 (1992); Erratum, Biophys. Chem. 45, 194 (1992).

[42] E.P. Petrov, in: Proc. Vith Int. Conf. on Methods and Applications of Fluorescence Spectroscopy, Berlin 1997, Köster, Berlin 1997, Paper P142.

[43] J.M.G. Martinho, A.L. Maçanita, M.N. Berberan-Santos, J. Chem. Phys. 90, 53 (1989).

[44] R.R. Alfano, S.L. Shapiro, W. Yu, Opt. Commun. 7, 191 (1973).

[45] Y.S. Liu, W.R. Ware, J. Phys. Chem. 97, 5980 (1993); 97, 5987 (1993); Y. Liu, P. de Mayo, W.R. Ware, J. Phys. Chem. 97, 5995 (1993).

[46] L.M. Bolot'ko, N.A. Borisevich, V.A. Tolkachev, Zh. Prikl. Spektrosk. 18, 974 (1973) [J. Appl. Spectrosc. (USSR) 18, 714 (1973)].

[47] K.M. Ho, C.T. Chan, C.M. Soukoulis, Phys. Rev. Lett. 65, 3152 (1990).

[48] E.N. Economou, M.M. Sigalas, Phys. Rev. B 48, 13434 (1993).

[49] R. Biswas, M.M. Sigalas, G. Subramania, K.-M. Ho, Phys. Rev. B 57, 3701 (1998).

[50] D.V. O'Connor, D. Phillips, Time-Correlated Single Photon Counting, Academic Press, London 1984.

[51] S.M. Barnett, R. Loudon, Phys. Rev. Lett. 77, 2444 (1996).

[52] T. Suzuki, P.K.L. Yu, J. Opt. Soc. Am. B 12, 570 (1995).

[53] D.S. Mogilevtsev, S.Ya. Kilin, in: Proc. European Quantum Electronics Conf., Hamburg 1996, IEEE, Piscataway (NJ) 1996, p. 219. 